\title{
Perspective de la plate-forme NEMOSIS dans le cadre d'une réduction de doses en imagerie
}

\author{
R. LAURENT ${ }^{1}$, R. GSCHWIND $^{1}$, M. SALOMON $^{2}$, J. HENRIET $^{1}$, L. MAKOVICKA $^{1}$
}

(Manuscrit reçu le 30 mars 2012, accepté le 2 juillet 2012)

RÉSUMÉ L'acquisition du mouvement est de plus en plus souvent effectuée pour améliorer la balistique des traitements en radiothérapie externe. Cependant, elle est source d'une exposition supplémentaire pour le patient. Le développement de la plate-forme de simulation numérique NEMOSIS (NEural NEtwork MOtion SImulation System) ouvre la voie à l'optimisation de la dose en imagerie. Elle permet de générer un mouvement pulmonaire localisé et personnalisé à partir du modèle 3D du patient. Pour 3 patients test, 5 à 6 points anatomiques ont été simulés puis comparés aux tracés du radiothérapeute. Dans le cas le plus défavorable, les résultats ont montré une précision moyennée sur l'ensemble des points d'un patient et sur toutes les phases d'environ $3 \mathrm{~mm}$ avec une incertitude élargie de tracé égale à $1,5 \mathrm{~mm}$ (intervalle de confiance de $95 \%$ ) et une incertitude maximale de phase atteignant $6,53 \mathrm{~mm}$. Une autre étude comparant les GTV (Gross Tumor Volume) d'un radiothérapeute et ceux calculés par NEMOSIS a été également menée. Un indice de Dice stipulant une correspondance minimale de 0,80 a été calculé entre les deux types de volumes. Ces résultats font de NEMOSIS un outil très prometteur en tant qu'alternative aux imageries irradiantes.

ABSTRACT Perspective of the NEMOSIS platform in the context of dose reduction in imaging. Motion acquisition is frequently performed to improve the treatment ballistics of external radiation therapy. Nevertheless, this acquisition is an additional source of radiation exposure for the patient. The development of the numerical simulation platform NEMOSIS (NEural NEtwork MOtion SImulation System) offers new perspectives for the dose optimization in imaging. It allows one to generate a localized and customized lung motion using a 3D model of the patient. For 3 patients, 5 to 6 anatomic points were simulated and compared with the points plotted by the radiation therapist. In the worst case, the results show an average accuracy of $3 \mathbf{~ m m}$ for all the points of a patient, at every phase, with an expanded uncertainty of plotting equal to $1.5 \mathrm{~mm}$ (confidence interval of $95 \%$ ) and a maximum uncertainty of $6.53 \mathrm{~mm}$ over one phase. Another study comparing the GTV (Gross Tumor Volume) of a radiation therapist and that computed by NEMOSIS was also carried out. A Dice index stating a minimum correspondence of 0.80 was computed between these two types of volumes. These results make NEMOSIS a very promising tool as an alternative solution to irradiation imaging.

Key words: lung motion / numerical simulation / dose optimization / imaging

\footnotetext{
IRMA/LCPR-AC/Chrono-Environnement, UMR 6249 CNRS, Université de Franche-Comté, BP 71427, 25211 Montbéliard Cedex, France.
}

2 AND/DISC/FEMTO-ST, UMR 6174 CNRS, Université de Franche-Comté, BP 527, 90016 Belfort Cedex, France. 


\section{Introduction}

La prise en compte des mouvements est un enjeu majeur dans l'acquisition de l'image pour améliorer leur qualité diagnostique. Étant donné la complexité de traiter tous les mouvements dans un même temps, nous nous sommes focalisés sur le mouvement pulmonaire. En effet, celui-ci est le mouvement le plus important en termes de variations, déformations et déplacements d'organes de la physiologie humaine. Dans le cas d'un traitement par radiothérapie externe des poumons, la connaissance et l'analyse de ce mouvement est primordial afin de traiter dans les meilleures conditions possibles chaque patient. Le tableau I présente le bilan des amplitudes de mouvements selon les différents axes et les différentes parties des poumons.

Actuellement, pour connaître ce mouvement, l'unique modalité utilisée en clinique permettant d'acquérir les mouvements respiratoires (incluant donc les mouvements pulmonaires) est le tomodensitomètre 4D (TDM). Son principe d'acquisition repose sur la synchronisation du signal respiratoire (Low et al., 2003 ; Rietzel et al., 2005) avec l'acquisition des images en 3 dimensions comme le résume la figure 1. Cette figure montre également en vert les instants irréguliers de la respiration. Bien que ces moments soient détectés ils participent néanmoins à la construction des images. Enfin les points tracés sur la courbe correspondent successivement aux phases $50 \%$ et $0 \%$ de la respiration, car dans l'hypothèse d'une respiration périodique, la phase (allant de 0 à $99 \%$ ) définit un instant précis de la respiration.

Lors du tri des images, chaque image est associée à une phase à l'aide du signal respiratoire. Du fait de l'irrégularité de ce signal (Fig. 1), il peut manquer des images à certaines phases. Pour éviter les images manquantes lors de la reconstruction d'un scanner 3D à une phase donnée, il existe une tolérance «temporelle» sur la sélection des phases. Ainsi, dans l'en-tête du fichier de l'image DICOM apparaît l'intervalle $\left(I_{P h}\right)$ en phase sur l'ensemble des coupes constituant l'image 3D. Cette tolérance est à l'origine des artefacts cinétiques présents sur les scans 3D. En effet, lorsqu'elle est trop élevée, certains tissus peuvent être visualisés deux fois, c'est-à-dire à deux positions différentes. Une étude effectuée par Yamamoto et al. (2008) sur une analyse de 50 TDM 4D a démontré la présence d'artefacts cinétiques aux environs du diaphragme ou du cœur sur $90 \%$ des images. La conséquence sur la mesure de la tumeur a été également identifiée : Sarker et al. (2010) ont corrélé des variations dans le volume pulmonaire en fonction de l'irrégularité du signal respiratoire lors de l'acquisition 4D. En outre, pour plusieurs inspirations maximales, ils ont mesuré, pour une position moyenne du centre de masse d'une tumeur, un écart-type de $1 \mathrm{~cm}$ pour une différence de 59,7\% en termes de volume pulmonaire. Par ailleurs, l'erreur est d'autant plus prononcée que la tumeur est de faible dimension. 
PERSPECTIVE DE LA PLATE-FORME NEMOSIS

\section{TABLEAU I}

Les mouvements pulmonaires dans la littérature.

The lung motions in the literature.

\begin{tabular}{|c|c|c|c|c|c|c|}
\hline \multirow{2}{*}{\multicolumn{2}{|c|}{ Localisation }} & \multicolumn{3}{|c|}{ Amplitudes } & \multirow[t]{2}{*}{ Nb. patient } & \multirow[t]{2}{*}{ Auteurs } \\
\hline & & $\mathbf{A P}$ & SI & GD & & \\
\hline \multicolumn{2}{|c|}{ Diaphragme } & & 10 à $35 \mathrm{~mm}$ & & 9,5 et 8 & $\begin{array}{l}\text { Davies et al. }(1994) \\
\text { Hanley et al. }(1999) \\
\text { Ford et al. }(2002)\end{array}$ \\
\hline \multirow[t]{3}{*}{ Lobes } & Supérieurs & $5,8 \mathrm{~mm}(5)$ & $8 \mathrm{~mm}(4,5)$ & & 20 & Giraud et al. (2001) \\
\hline & Moyen & & $6,75 \mathrm{~mm}$ & & 4 & Murphy et al. (2002) \\
\hline & Inférieurs & & 15,6 & 5 & 16 & $\begin{array}{l}\text { Van de Steene } \text { et al. } \\
\text { (1998) }\end{array}$ \\
\hline \multicolumn{2}{|c|}{$\begin{array}{l}\text { Toutes localisations } \\
\text { confondues }\end{array}$} & $<0,82 \mathrm{~cm}$ & $<1,86 \mathrm{~cm}$ & $<0,71 \mathrm{~cm}$ & 20 & $\begin{array}{l}\text { Redmond et al. } \\
(2009)\end{array}$ \\
\hline \multicolumn{2}{|c|}{$\begin{array}{l}\text { Toutes localisations } \\
\text { confondues }\end{array}$} & $<0,59 \mathrm{~cm}$ & $<1,34 \mathrm{~cm}$ & $<0,4 \mathrm{~cm}$ & 166 & Liu et al. (2007) \\
\hline
\end{tabular}

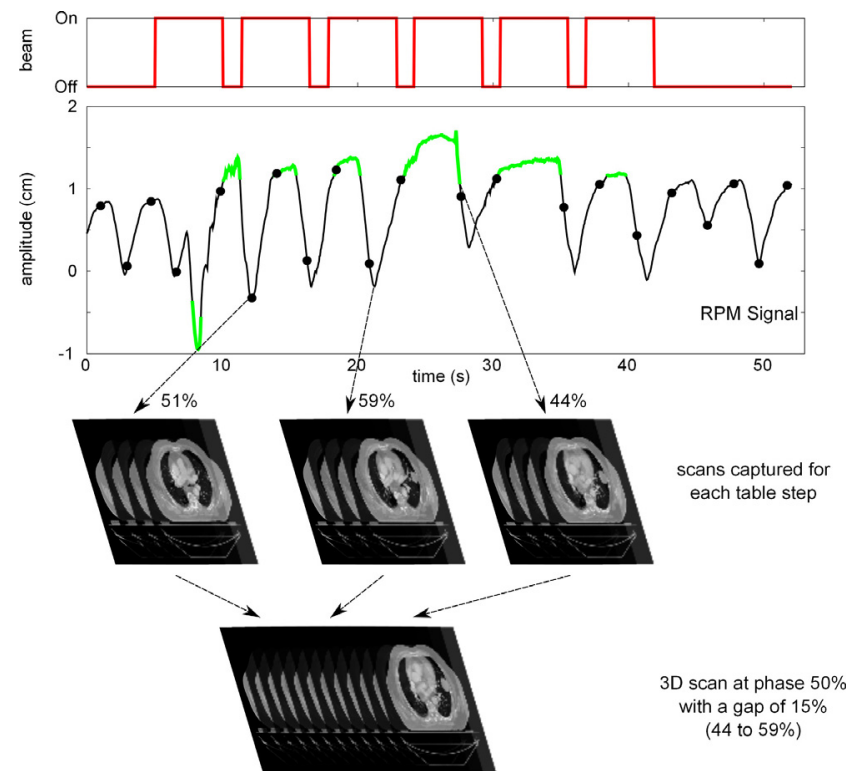

Figure 1 - Principe d'acquisition d'un scanner $4 D$.

Acquisition principle of a $4 \mathrm{DCT}$. 
En plus des artefacts cinétiques, le temps de rotation du tube RX est à l'origine d'un autre artefact appelé flou cinétique. Alors que les artefacts cinétiques sont définis dans la direction Supéro-Inférieur (du fait de l'acquisition séquentielle des coupes), les flous cinétiques sont caractérisés dans le plan axial (i.e. dans une coupe). Ils correspondent au mouvement effectué pendant le temps de rotation. Ainsi, plus ce temps est court, moins les images sont sujettes aux flous cinétiques, au détriment du contraste. Les artefacts résultants conduisent à une mauvaise définition des structures anatomiques du fait qu'elles peuvent être présentes plusieurs fois. Cet artefact n'est pas négligeable. En effet, Persson et al. (2010) notent, en plus des artefacts cinétiques, l'influence des flous cinétiques sur des tracés de GTV (Gross Tumor Volume) : ces derniers produisant alors un effet de volume partiel sur la tumeur. La somme de ces deux effets induit des différences de volumes GTV allant jusqu'à $90 \%$.

Étant donné que l'un des problèmes majeurs de l'acquisition est la reconstruction des images, une des perspectives pour améliorer la connaissance du mouvement consiste à élaborer des techniques d'analyse des images afin d'orienter la reconstruction. Dans cette optique de réduction des artefacts cinétiques, Ehrhardt et al. (2007) proposent une méthode fondée sur les mouvements apparents pour l'amélioration de la reconstruction 4D des images. Pour cela, ils déterminent un champ de vecteurs à partir de deux scans proches de la phase souhaitée en utilisant un algorithme de recalage non-linéaire. Ce champ de vecteurs est ensuite utilisé lors de la reconstruction des images en interpolant les données à chaque phase exacte souhaitée. Zeng et al. (2008) propose une nouvelle méthode de tri de données 4D : celle-ci, au lieu d'être fondée sur le signal respiratoire, est une méthode itérative analysant le mouvement anatomique interne. Pour ce faire, leur algorithme étudie les variations de volume des organes thoraciques pour ordonner les images. Sur 5 données de patient testées, 3 ont fourni des images similaires à celles proposées par l'analyse du signal respiratoire, les 2 autres ont présenté moins d'artefacts. D'autres travaux ont suivi cette voie pour améliorer le classement (Johnston et al., 2011).

Cette liste de travaux permet de mettre en avant le fait que quelle que soit la méthode de reconstruction utilisée, elle est toujours dépendante de la qualité des images et de la régularité de la respiration du patient lors de l'acquisition. Comme nous l'avons vu à travers la figure 1 , cette reproductibilité est difficile : le niveau de stress du patient n'est pas une donnée quantifiable ni maîtrisable.

Pour améliorer la qualité de l'information relative au mouvement, d'autres voies de recherche ont été étudiées notamment au niveau de l'emploi des méthodes numériques. Ces méthodes sont classées selon deux catégories : les estimations et les simulations. La simulation consiste principalement à prédire les autres états en 
TABLEAU II

Résumé de l'ensemble des méthodes de simulation et d'estimation. Summary of existing simulation and estimation techniques.

\begin{tabular}{|c|c|c|c|c|}
\hline Type & Méthodes & Temps de calcul & Précision & Auteurs \\
\hline \multirow[t]{4}{*}{ Simulation } & $\begin{array}{l}\text { Mécanique des milieux } \\
\text { continus }\end{array}$ & $\begin{array}{c}\text { Quelques } \\
\text { dizaines } \\
\text { de minutes à } \\
\text { plusieurs heures }\end{array}$ & $\begin{array}{c}\text { Inférieure } \\
\text { à la résolution } \\
\text { de la méthode }\end{array}$ & Villard (2006) \\
\hline & $\begin{array}{l}\text { Marqueurs externes } \\
\text { \& modèle 3D }\end{array}$ & Temps réel & $\begin{array}{l}\text { Supérieure à } 3 \mathrm{~mm} \\
\text { (uniquement les } \\
\text { contours) }\end{array}$ & $\begin{array}{l}\text { Hostettler et al. (2006, } \\
\text { 2008) }\end{array}$ \\
\hline & Morphing & $\begin{array}{l}\text { Plusieurs } \\
\text { dizaines de } \\
\text { secondes }\end{array}$ & $\begin{array}{c}\text { Pas de mesure } \\
\text { possible(uniquement } \\
\text { les contours) }\end{array}$ & Laurent et al. (2010) \\
\hline & $\begin{array}{c}\text { Relation pression- } \\
\text { volume \& analyse par } \\
\text { éléments finis }\end{array}$ & Inconnu & $\begin{array}{c}\text { Écart moyen } \\
\text { inférieur à 4,5 mm }\end{array}$ & Eom et al. (2010) \\
\hline \multirow[t]{3}{*}{ Estimation } & Recalage déformable & $\begin{array}{l}\text { Une à plusieurs } \\
\text { dizaines de } \\
\text { secondes }\end{array}$ & $\begin{array}{c}\text { Inférieure } \\
\text { à la résolution } \\
\text { de la méthode }\end{array}$ & $\begin{array}{l}\text { Boldea (Yang et al., } \\
\text { 2008 ; Boldea, 2006) }\end{array}$ \\
\hline & Modélisation 5D & Inconnu & $\begin{array}{l}\text { Écart moyen } \\
\text { inférieur au } \\
\text { millimètre }\end{array}$ & $\begin{array}{l}\text { Low et al. (Zhao et al., } \\
2009 \text {; Low et al., 2005) }\end{array}$ \\
\hline & PoPi-model & $\begin{array}{l}\text { Quelques } \\
\text { secondes }\end{array}$ & Inférieure à $3 \mathrm{~mm}$ & $\begin{array}{l}\text { Vandemeulebroucke } \\
\text { et al. }(2007,2011)\end{array}$ \\
\hline
\end{tabular}

fonction du temps à partir d'un état initial, alors que l'estimation consiste à insérer des connaissances a priori sur un modèle mathématique - connaissances apportées généralement par la mesure même du mouvement.

Le tableau II fait le bilan des méthodes numériques avec leurs performances. Comme nous pouvons le voir, les estimateurs sont plus précis que les simulateurs. En effet, l'apport des connaissances a priori (provenant du scanner 4D) permet d'affiner avec précision les modèles mathématiques en place. Cependant leur inconvénient est justement le besoin de recourir à cette modalité très irradiante. Les simulateurs ont, quant à eux, des résultats variables qui n'offrent pas de réelle réponse mêlant précision (interne et pariétale) avec un temps de calcul minimal pour envisager leurs applications en routine clinique.

L'analyse de ces différentes méthodes permet de mettre en avant les points importants pour la mise en œuvre de notre simulation du mouvement pulmonaire. En effet, elle doit être véloce, personnalisée, précise, non artefactée et apporter un gain pour le patient en termes de confort (aucune nécessité de mesure contraignante ou de blocage respiratoire) et de radioprotection. C'est la raison pour laquelle nous avons opté pour les réseaux de neurones artificiels (RNA). 


\section{Matériels et méthodes}

Un neurone artificiel est une analogie à un réseau de neurones biologiques. Tout comme eux, ils possèdent une ou plusieurs entrées et une ou plusieurs sorties. Montés en réseau, les neurones permettent l'interpolation de phénomènes complexes sous condition d'avoir effectué un apprentissage au préalable.

Le réseau de neurones artificiels que nous allons considérer est une version optimisée de notre plate-forme de simulation du mouvement pulmonaire personnalisé (Laurent et al., 2011, 2012) nommée NEMOSIS (NEural NEtwork MOtion SImulation System). L'optimisation a consisté à simplifier les pré-requis pour l'utilisation de la méthode (5 entrées au lieu de 8). Ainsi, au lieu de spécifier un vecteur de déplacement, on définit les coordonnées d'un point à simuler. Les 5 entrées sont donc les coordonnées $X_{0} \%, Y_{0} \%$ et $Z_{0} \%$ du point, le volume du patient et la phase à laquelle on souhaite déterminer la nouvelle position.

La topologie d'un réseau de neurones dépend à la fois du nombre d'entrées et de sorties mais également du jeu de données d'apprentissage. Le nombre d'entrées étant modifié, la nouvelle topologie a été évaluée en nous basant sur les processus d'optimisation décrits dans notre étude antérieure (Laurent et al., 2012). Les résultats ont montré que le nombre maximal de neurones à considérer est de 5 . Cette topologie sera conservée pour l'ensemble des résultats présentés.

\section{Jeu de données}

Pour cette application clinique, en plus des 5 patients constituant le jeu de données, 3 patients disposant de TDM 4D focalisé sur la tumeur ont été sélectionnés selon les critères décrits dans nos travaux (Laurent et al., 2012). Pour chaque patient, 10 phases respiratoires sont disponibles. Les voxels ont pour dimensions $0,98 \times$ $0,98 \times 2,5 \mathrm{~mm}^{3}$. Les volumes pulmonaires ont été définis à l'aide des scanners $3 \mathrm{D}$ thorax. Le tableau III résume les informations relatives à ces nouveaux patients qui constituent notre jeu de données de test.

Afin de quantifier la performance de notre méthode, nous avons comparé des mouvements de points simulés par NEMOSIS avec ceux tracés sur les TDM 4D. Aussi, comme le montre le tableau III, 5 à 6 points ont été définis par patient de test. Ceux-ci ont été marqués conformément au protocole décrit dans (Laurent et al., 2011, 2012) et ont été choisis dans des régions présentant le moins de flous et d'artefacts cinétiques, afin de réduire les erreurs lors du tracé du médecin.

En complément, pour décrire le cas d'une application en routine clinique de NEMOSIS, nous avons demandé au radiothérapeute de tracer sur l'ensemble des phases les contours tumoraux. Seuls les GTV ont été tracés, car d'après les 
TABLEAU III

Informations relatives aux données patient utilisées pour l'étude clinique de NEMOSIS. Information related to the patient data used for the clinical study of NEMOSIS.

\begin{tabular}{cccc}
\hline & \multicolumn{3}{c}{ Patient de test } \\
\cline { 2 - 4 } & $\mathbf{1}$ & $\mathbf{2}$ & $\mathbf{3}$ \\
\hline Nb. de coupes & 40 & 48 & 32 \\
\hline Volume pulmonaire $(l)$ & 4,04 & 3,10 & 4,42 \\
\hline Nb. de points anatomiques & 5 & 6 & 5 \\
Nb. de points GTV & 264 & 222 & 519 \\
Écarts max. de points & 30 & 41 & 59 \\
\hline
\end{tabular}

protocoles cliniques, les autres contours ne sont pas « dessinés » par la main du médecin mais calculés : par exemple, la définition du CTV (Clinical Target Volume) sera effectuée en appliquant une marge dépendante de la pathologie à traiter autour du GTV. Cependant, pour éprouver au maximum notre algorithme, nous avons demandé que les GTV soient tracés sur les images MIP (Maximal Intensity Pixel). Les images MIP sont générées par comparaison et sélection des pixels ayant la plus grande unité de Hounsfield sur l'ensemble des phases d'un scanner 4D. Ces images sont particulièrement bien adaptées pour les tumeurs pulmonaires car le contraste entre les tissus sains et tumoraux est important. Elles permettent ainsi de déterminer sur une seule image 3D, les marges relatives à l'amplitude du mouvement de la tumeur.

La tableau III montre le nombre de points définissant le GTV à la phase $0 \%$ pour chaque patient. Seul ce nombre est utile car ces points seront «injectés » comme entrées du RNA. Néanmoins, il est important de noter la variation des tracés par le radiothérapeute (présentée sur la dernière ligne du Tab. III). Plus connue sous le terme de reproductibilité intra-opérateur (Louie et al., 2010), cette dernière peut être quantifiée par la variation du nombre de points définissant le GTV ou encore par le volume défini par les contours. L'acquisition des images à différents instants peuvent expliquer ces variations.

\section{Validation}

La validation de notre algorithme a nécessité une étude sur les incertitudes. Cellesci se distinguent, dans le cas d'un TDM 4D, selon deux catégories : les incertitudes de tracé et les incertitudes de phase.

L'incertitude de tracé $\left(u_{T r}\right)$ est une incertitude-type sur la mesure (incertitude de type B). Celle-ci est estimée par la résolution spatiale du système (exprimée en millimètre). Les points étant tracés dans les images tomodensitométriques, 
l'estimation maximale de $u_{T r}$ consiste donc à considérer uniquement sa plus grande valeur selon les 3 dimensions. Étant donné que la probabilité de tracer un point au sein d'un voxel est égale dans tout son volume, $u_{T r}$ s'écrit pour cette distribution rectangulaire :

$$
u_{T r}=k \frac{\max \left(R_{i}\right)}{2 \sqrt{3}}
$$

où $R_{i}$ est la résolution spatiale (définie par la dimension des voxels $0,98 \times 0,98 \times$ $2,5 \mathrm{~mm}^{3}$ ) suivant la dimension $i$ et $k$ est le facteur d'élargissement ( $k=1$ pour un intervalle de confiance de $68 \%$ ).

Afin de considérer un intervalle de confiance plus important que celui de cette incertitude-type, nous utiliserons par la suite l'incertitude élargie, valant 1,5 mm dans notre étude, pour un intervalle de confiance de $95 \%(k=2)$.

L'incertitude de phase $\left(u_{P h}\right)$ ne peut pas être quantifiée car elle impliquerait de connaître le mouvement recherché. Néanmoins, pour l'évaluer, nous pouvons comparer les données acquises à un interpolateur, ici NEMOSIS.

Dans l'optique de mesurer l'incertitude maximale, nous avons choisi l'axe où $u_{T r}$ est maximale : l'axe Supéro-Inférieur (SI). Ainsi, le tableau IV résume les incertitudes maximales de phase obtenues en appliquant la valeur absolue sur la différence de la coordonnée tracée sur le scanner $4 \mathrm{D}\left(z_{4 D}\right)$ et la plus éloignée de cette valeur calculée par NEMOSIS $\left(z_{N E M O S I S}\right)$ dans l'intervalle $I_{P h}$ de phase donné :

$$
u_{P h}= \pm \max \left|z_{4 \mathrm{D}}-\mathrm{z}_{\mathrm{NEMOSIS}}\right|_{\mathrm{I}_{\mathrm{Ph}}} .
$$

La validation des contours tumoraux a consisté à évaluer la performance de l'application de NEMOSIS sur les GTV par comparaison des volumes tumoraux simulés et tracés par le radiothérapeute pour chaque phase respiratoire. De plus, le GTV maximal induit par l'ensemble des GTV calculés sur les phases a été déterminé, puis analysé avec le GTV MIP.

\section{Résultats}

Les temps de calcul de NEMOSIS sont extrêmement faibles. La simulation des 100 phases des points anatomiques de chacun de ces patients nécessite de 50 à $80 \mathrm{~ms}$. En ce qui concerne le calcul des GTV sur les 100 phases, celui-ci s'est 
TABLEAU IV

Définition des intervalles $\left(I_{P h}\right)$ et incertitudes $\left(u_{P h}\right)$ de phases pour chaque patient de test. Definition of the phase intervals $\left(I_{P h}\right)$ and uncertainties $\left(u_{P h}\right)$ for each test patient.

\begin{tabular}{ccccccc}
\hline & \multicolumn{2}{c}{ Patient de test } & & \multicolumn{2}{c}{$\mathbf{3}$} \\
\cline { 2 - 7 } Phase $(\%)$ & $I_{P h}(\%)$ & $\begin{array}{c}u_{P h}(\mathrm{~mm}) \\
\text { point } 3)\end{array}$ & $I_{P h}(\%)$ & $\begin{array}{c}u_{p h}(\mathrm{~mm}) \\
\text { point } 0)\end{array}$ & $I_{P h}(\%)$ & $\begin{array}{c}u_{P h}(\mathrm{~mm}) \\
\text { point } 2)\end{array}$ \\
\hline 0 & {$[82 ; 10]$} & 1,8 & {$[96 ; 6]$} & 0,8 & {$[97 ; 2]$} & 0,9 \\
10 & {$[94 ; 21]$} & 2,5 & {$[6 ; 14]$} & 0,9 & {$[6 ; 13]$} & 0,7 \\
20 & {$[21 ; 43]$} & 6,5 & {$[16 ; 23]$} & 1,5 & {$[16 ; 23]$} & 1,7 \\
30 & {$[30 ; 43]$} & 4,0 & {$[27 ; 34]$} & 3,2 & {$[23 ; 34]$} & 1,3 \\
40 & {$[41 ; 43]$} & 1,5 & {$[36 ; 46]$} & 2,2 & {$[37 ; 44]$} & 2,0 \\
50 & {$[41 ; 55]$} & 1,7 & {$[46 ; 55]$} & 1,4 & {$[46 ; 54]$} & 0,6 \\
60 & {$[55 ; 60]$} & 1,7 & {$[54 ; 64]$} & 1,9 & {$[56 ; 63]$} & 1,4 \\
70 & {$[68 ; 71]$} & 0,9 & {$[64 ; 75]$} & 2,9 & {$[66 ; 74]$} & 3,5 \\
80 & {$[79 ; 83]$} & 0,3 & {$[74 ; 84]$} & 1,1 & {$[80 ; 85]$} & 0,6 \\
90 & {$[79 ; 94]$} & 2,8 & {$[87 ; 95]$} & 0,9 & {$[87 ; 92]$} & 0,5 \\
\hline
\end{tabular}

déroulé sur un intervalle de temps allant de $600 \mathrm{~ms}$ à $1,2 \mathrm{~s}$. Par déduction, notre réseau est capable de déterminer un contour tumoral sur une phase en 6 à 12 ms.

\section{Points anatomiques}

La figure 2 présente un point pour chaque patient testé, mais uniquement sur l'axe ' $z$ ': les autres axes présentant un mouvement peu prononcé n'apportent pas d'information supplémentaire. Les figures $2 b$ et $2 c$ illustrent une réduction des amplitudes maximales alors que la figure 2a montre, quant à elle, une simulation de la position à la phase $50 \%$ au-delà de la valeur mesurée. Ceci résulte des mouvements des patients ayant servis à l'apprentissage de NEMOSIS : ces patients ont présenté sur des points similaires, suivant les cas, un mouvement plus ou moins important que celui mesuré sur ces patients.

La figure 2c montre un défaut dans la continuité entre les phases 99 et $0 \%$ simulées par NEMOSIS. Pour le moment l'apprentissage du mouvement a été réalisé à partir de 5 patients : même si les résultats sont déjà intéressants, cela est insuffisant. L'augmentation du jeu de données, en points et en patients, permettra de pallier ces « défauts » que mettent en évidence nos résultats. 

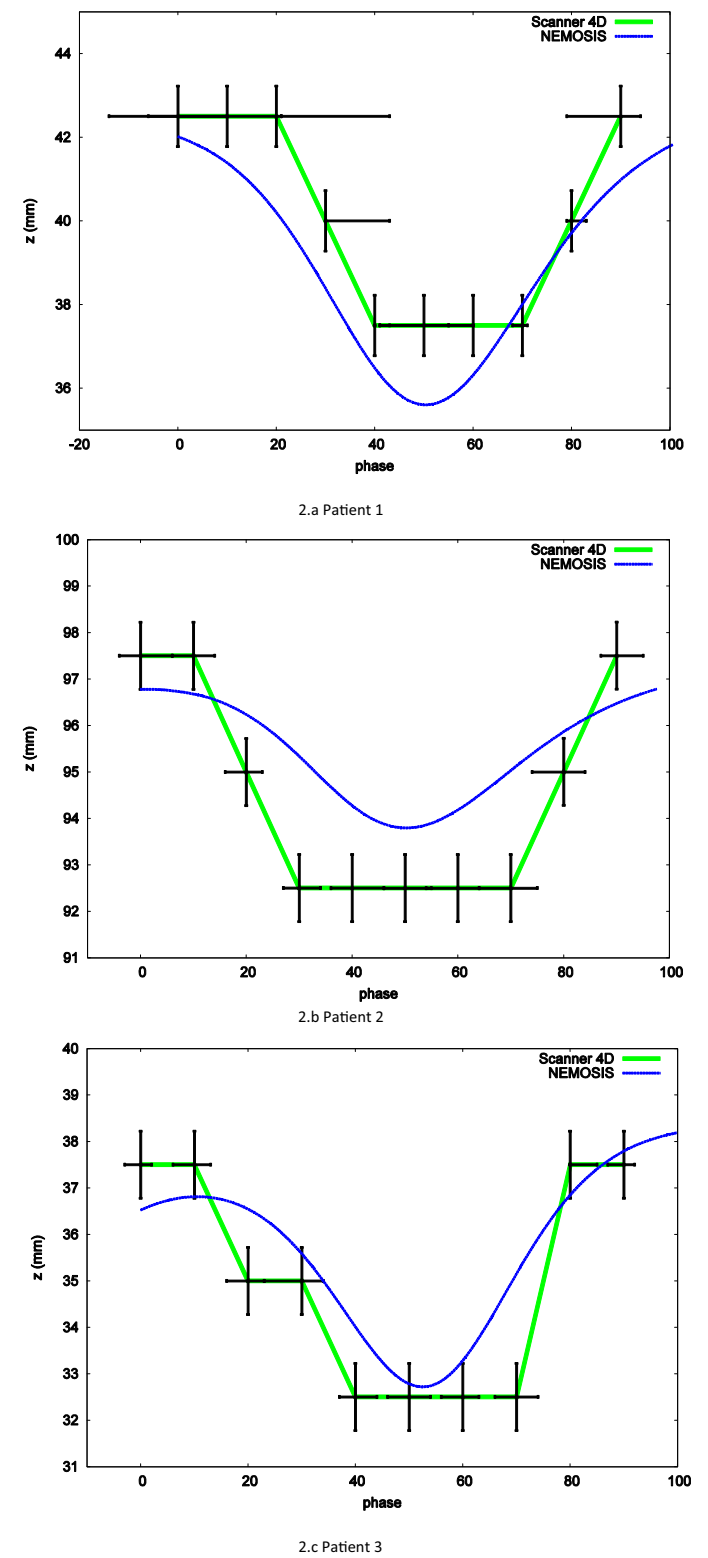

Figure 2 - Mouvement respectif d'un point du patient 1, 2 et 3 sur l'axe Supéro-Inférieur. (a) Patient 1, (b) Patient 2, (c) Patient 3.

Motion of a point for, respectively, patient 1, 2 and 3 along the Supero-Inferior axis. (a) Patient 1, (b) Patient 2, (c) Patient 3. 
TABLEAU V

Écarts moyens (écarts-types) en mm entre les points interpolés et tracés. Mean deviation (standard deviation) in $\mathbf{~ m m}$ between interpolated and plotted points.

\begin{tabular}{cccc}
\hline Phase $(\%)$ & \multicolumn{3}{l}{ Patient de test } \\
\cline { 2 - 4 } & $\mathbf{1}$ & $\mathbf{2}$ & $\mathbf{3}$ \\
\hline 0 & $0,61( \pm 0,37)$ & $0,56( \pm 0,40)$ & $0,84( \pm 0,34)$ \\
10 & $0,92( \pm 0,41)$ & $1,10( \pm 0,71)$ & $1,76( \pm 0,59)$ \\
20 & $2,51( \pm 0,45)$ & $2,20( \pm 0,74)$ & $1,91( \pm 0,52)$ \\
30 & $3,86( \pm 2,62)$ & $3,32( \pm 0,47)$ & $2,69( \pm 1,41)$ \\
40 & $4,91( \pm 2,30)$ & $2,80( \pm 0,98)$ & $2,59( \pm 0,86)$ \\
50 & $5,22( \pm 2,05)$ & $2,34( \pm 1,23)$ & $2,96( \pm 0,81)$ \\
60 & $4,47( \pm 1,99)$ & $2,14( \pm 1,19)$ & $2,96( \pm 1,34)$ \\
70 & $2,76( \pm 1,19)$ & $2,37( \pm 1,18)$ & $2,67( \pm 1,85)$ \\
80 & $2,16( \pm 0,66)$ & $1,99( \pm 0,98)$ & $2,08( \pm 1,29)$ \\
90 & $1,80( \pm 0,39)$ & $0,69( \pm 0,35)$ & $1,59( \pm 1,16)$ \\
\hline Moyenne $(\sigma)$ & $2,92( \pm 2,16)$ & $1,95( \pm 1,23)$ & $2,20( \pm 1,29)$ \\
\hline
\end{tabular}

Le tableau $\mathrm{V}$ présente les écarts moyens sur tous les points et à toutes les phases pour les 3 patients test.

Comme stipulé auparavant, le tableau IV renseigne les incertitudes de phase $\left(u_{P h}\right)$ pour les points présentés par la figure 2 . Il montre que les incertitudes les plus élevées sont sur les gradients de mouvement les plus importants. Dans le cas du patient 1 , les phases de 0 à $30 \%$ ayant un $I_{P h}$ élevé, impliquent de grandes incertitudes. NEMOSIS, utilisant seulement la phase $0 \%$ en entrée, a alors répercuté ces biais.

L'analyse de la précision des résultats est donc délicate puisque l'ensemble des incertitudes doit être considéré. Il faut donc pondérer chaque écart moyen du tableau $\mathrm{V}$ avec à la fois les incertitudes de phase $u_{P h}$ du tableau IV et les incertitudes élargies de tracé $u_{T r}$. En se plaçant dans le cas le plus défavorable, c'est-à-dire en additionnant les incertitudes, la majeure partie des écarts entre NEMOSIS et le scanner 4D est inférieure à cette somme.

\section{Contours tumoraux}

Pour estimer la capacité de NEMOSIS à reproduire des contours tumoraux en fonction du mouvement pulmonaire, 10 GTV et le GTV MIP ont été tracés pour les 3 patients test. Ceux-ci présentent des tumeurs pariétales ou médiastinales. Dans ce dernier cas, il est particulièrement délicat pour le radiothérapeute d'être reproductible dans son tracé (voir Tab. III). 
TABLEAU VI

Volume tumoral (GTV) en $\mathrm{cm}^{3}$ déterminé par les contours du radiothérapeute et de NEMOSIS pour chaque patient test.

Tumor volume (GTV) in $\mathrm{cm}^{3}$ determined by the contours given by the radiotherapist and by NEMOSIS for each test patient.

\begin{tabular}{|c|c|c|c|c|c|c|c|c|c|c|c|c|}
\hline \multirow[b]{3}{*}{$\begin{array}{c}\text { Phase } \\
(\%)\end{array}$} & \multicolumn{12}{|c|}{ Patient } \\
\hline & 1 & 2 & 3 & 1 & 2 & 3 & 1 & 2 & 3 & 1 & 2 & 3 \\
\hline & \multicolumn{3}{|c|}{$V_{\text {Med }}$} & \multicolumn{3}{|c|}{$V_{N E M}$} & \multicolumn{3}{|c|}{$V_{M e d} \cap V_{N E M}$} & \multicolumn{3}{|c|}{$D\left(V_{M e d}, V_{N E M}\right)$} \\
\hline 0 & 44,46 & 20,75 & 104,22 & 41,02 & 18,88 & 104,88 & 40,10 & 18,16 & 101,77 & 0,9382 & 0,9165 & 0,9734 \\
\hline 10 & 43,53 & 19,25 & 97,64 & 40,75 & 19,20 & 101,91 & 39,22 & 16,27 & 87,58 & 0,9307 & 0,8463 & 0,8778 \\
\hline 20 & 44,40 & 19,59 & 116,64 & 40,67 & 19,71 & 102,18 & 38,70 & 16,46 & 91,01 & 0,9098 & 0,8377 & 0,8318 \\
\hline 30 & 45,14 & 19,79 & 118,96 & 40,93 & 19,60 & 100,68 & 38,80 & 15,84 & 91,68 & 0,9016 & 0,8043 & 0,8348 \\
\hline 40 & 45,06 & 19,52 & 112,79 & 40,72 & 19,51 & 100,54 & 38,39 & 15,56 & 87,87 & 0,8951 & 0,7973 & 0,8238 \\
\hline 50 & 44,11 & 19,31 & 115,08 & 40,30 & 20,77 & 98,98 & 36,70 & 17,45 & 87,26 & 0,8696 & 0,8708 & 0,8153 \\
\hline 60 & 45,95 & 19,24 & 115,37 & 41,31 & 20,77 & 98,54 & 37,14 & 17,87 & 86,14 & 0,8512 & 0,8933 & 0,8054 \\
\hline 70 & 44,85 & 18,93 & 119,35 & 42,22 & 20,77 & 100,85 & 38,53 & 17,96 & 89,00 & 0,8850 & 0,9048 & 0,8083 \\
\hline 80 & 43,69 & 19,09 & 123,72 & 42,08 & 20,77 & 102,02 & 39,31 & 18,13 & 90,17 & 0,9166 & 0,9097 & 0,7989 \\
\hline 90 & 43,70 & 19,33 & 117,56 & 40,92 & 20,77 & 100,61 & 39,07 & 18,18 & 87,79 & 0,9234 & 0,9067 & 0,8048 \\
\hline MIP & 49,27 & 22,05 & 150,51 & 44,10 & 21,66 & 124,65 & 41,80 & 19,01 & 114,86 & 0,8954 & 0,8698 & 0,8348 \\
\hline
\end{tabular}

Pour les patients 1,2 et 3 , la figure 3 montre une coupe prise à la phase $30 \%$ avec une partie visible de la tumeur. Les coupes sélectionnées ont été choisies pour des raisons de lisibilité des différents contours : en effet, sur certaines coupes les contours se superposent rigoureusement. La tumeur du patient 2 (Fig. 3b) est située dans la partie supérieure du poumon. Cette zone dispose de peu de points pour l'apprentissage et le calcul. NEMOSIS ne simule pas ici le mouvement de façon réaliste (Tab. VI). Néanmoins, l'analyse de la figure 3 montre que les contours simulés sont proches de ceux tracés par le radiothérapeute, les différences s'expliquant principalement par l'erreur intra-opérateur.

Pour vérifier la pertinence des contours, nous avons étudié le comportement des volumes tumoraux en fonction du temps (Tab. VI). Ce tableau référence trois types de volume: le volume tumoral déterminé par les contours du médecin $\left(V_{M e d}\right)$, le volume tumoral simulé par NEMOSIS $\left(V_{N E M}\right)$ et leur volume en commun $\left(V_{\text {Med }} \cap V_{N E M}\right)$. Afin de comparer plus intuitivement les volumes communs, la dernière colonne donne l'indice de DICE entre $V_{M e d}$ et $V_{N E M}$. Cet indice s'exprime sous la forme générale :

$$
D(A, B)=\frac{\operatorname{card}(A \cap B)}{\alpha \operatorname{card}(A)+(1-\alpha) \operatorname{card}(B)} .
$$




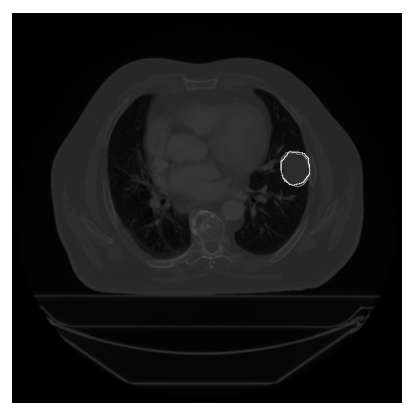

3.a.1 Coupe du patient 1
3.a.1 Slice from patient 1

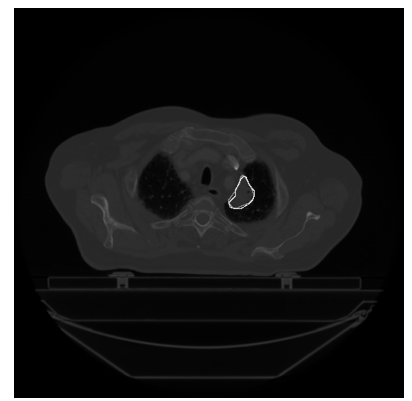

3.b. 1 Coupe du patient 2

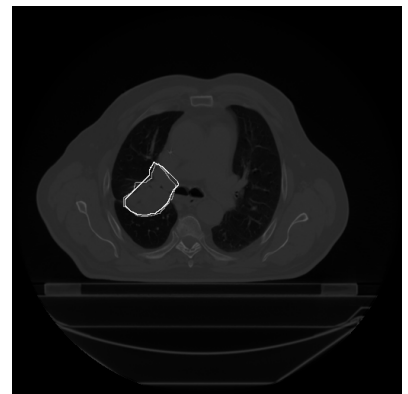

3.c.1 Coupe du patient 3

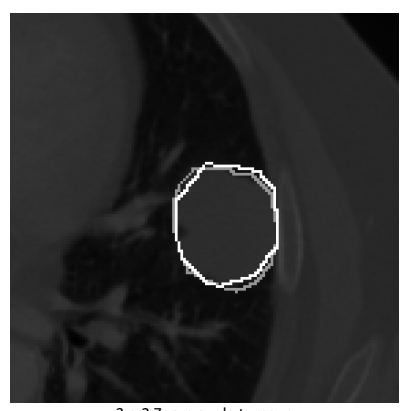

3.a.2 Zoom sur la tumeur
3.a.2 Zoom on the tumor

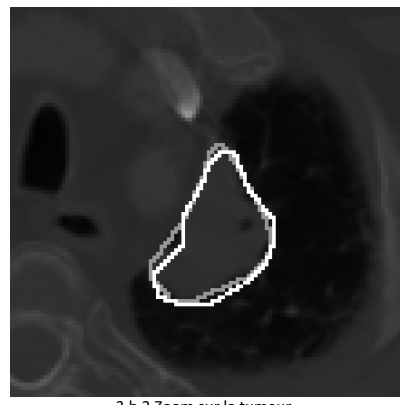

3.b.2 Zoom sur la tumeu

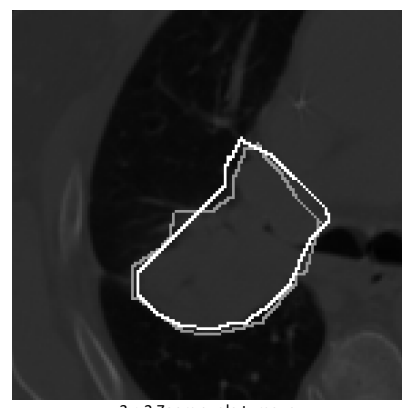

3.c. 2 Zoom sur la tumeur

Figure 3 - Contours tumoraux sur une coupe des patients 1,2 et 3 pour la phase $30 \%$. En gris les contours du radiothérapeute et en blanc les contours de NEMOSIS. (a) Coupe du patient 1 ; (b) coupe du patient 2 ; (c) coupe du patient 3 ; (d) zoom sur tumeur patient 1 ; (e) zoom sur tumeur patient $2 ;(f)$ zoom sur tumeur patient 3.

Tumor contours over one slice from the patients 1, 2 and 3 for the 30\% phase. In gray, the contours given by the radiotherapist and in white by NEMOSIS. (a) Slice from patient 1; (b) slice from patient 2; (c) slice from patient 3; (d) zoom on the tumor patient 1; (e) zoom on the tumor patient $2 ;(f)$ zoom on the tumor patient 3. 
Dans le cas d'une comparaison d'ensembles censés identiques, $\alpha$ prend alors la valeur de $\frac{1}{2}$. La relation devient alors :

$$
D(A, B)=\frac{2 \operatorname{card}(A \cap B)}{\operatorname{card}(A)+\operatorname{card}(B)} .
$$

Dans notre contexte, cela donne donc :

$$
D\left(V_{\text {Med }}, V_{N E M}\right)=\frac{2 \operatorname{card}\left(V_{M e d} \cap V_{N E M}\right)}{\operatorname{card}\left(V_{M e d}\right)+\operatorname{card}\left(V_{N E M}\right)} .
$$

Ainsi, plus $V_{M e d}$ et $V_{N e m}$ seront identiques plus tendra vers 1.

En premier lieu, nous pouvons voir que la mesure des volumes ne montre pas de variation identifiable en fonction du temps. Cependant, le volume mesuré au MIP est bien supérieur au volume identifié à chaque phase, ce qui implique l'existence d'un mouvement. Ainsi, dans cette première approche, nous pouvons déduire, en pondérant néanmoins par les incertitudes de reproductibilité des tracés, de résolutions spatiales et de phases, qu'une tumeur semble être de nature incompressible.

En second lieu, le calcul de NEMOSIS est basé sur le volume à la phase $0 \%$. Pour les patients 1 et 3 , notre méthode simule une diminution, bien que faible, du volume tumoral vers la phase $50 \%$ avant de tendre vers les valeurs initiales. Cela signifie donc que notre algorithme a bien considéré des mouvements différents en fonction des points: dans l'axe crânio-caudal, les points tendant vers le diaphragme ont présenté une variation plus importante que ceux tendant vers la partie supérieure des poumons. Comme il a été expliqué précédemment, les résultats pour le patient 2 ne sont pas à considérer au niveau de la prise en compte du mouvement, l'apprentissage étant insuffisant dans cette région du poumon: après la phase $50 \%$, plus aucun mouvement n'est simulé.

Ensuite, l'analyse des deux dernières colonnes du tableau VI montre que, dans l'ensemble, l'indice de DICE appliqué à $V_{M e d}$ et $V_{N E M}$ est supérieur à 0,85 pour les patients 1 et 2 . Les difficultés de contourage de la tumeur sur le patient 3 font que les indices sont moindres, tout en étant supérieurs à 0,80 dans la majorité des cas : NEMOSIS reproduit le contour de la phase $0 \%$ en fonction du temps alors que le radiothérapeute dessine un nouveau tracé à chaque phase.

Pour finir, les volumes mesurés sur les MIP sont supérieurs aux volumes mesurés sur les phases, ce qui signifie donc que NEMOSIS reproduit le mouvement de façon cohérente. 


\section{Discussion}

Nous constatons d'après le tableau VI que NEMOSIS ne restitue pas exactement les données du radiothérapeute pour la phase $0 \%$. Ainsi, même lorsque le volume est identique (patient 3), l'indice de DICE n'est pas de $100 \%$ et la superposition des contours présente des biais. Les différences au niveau des volumes tumoraux tiennent aussi aux images des patients. En effet, les coupes extrémales montrent généralement la tumeur sous un effet de volume partiel. Cet effet modifie les intensités des voxels, ce qui perturbe le tracé des contours par le radiothérapeute.

Il est important de rappeler que NEMOSIS a appris le mouvement sur des tissus sains. De plus, l'expérience du médecin montre que le mouvement d'une tumeur juxtaposée à la paroi ne présente pas les amplitudes attendues par rapport à sa localisation : celles-ci sont inférieures. Ces considérations nous donnent des pistes d'amélioration de notre algorithme.

Par ailleurs, NEMOSIS utilise actuellement les coordonnées des points définis à la phase $0 \%$, bien que son objectif soit de supprimer l'utilisation du scanner 4D. À l'heure actuelle, aucun protocole n'est en vigueur pour synchroniser un signal respiratoire avec une acquisition TDM 3D en respiration libre. Cependant, d'après le tableau IV, nous avons vu qu'il était possible de connaître les phases de chacune des coupes définissant une image 3D. Une solution serait d'inclure une synchronisation respiratoire à l'acquisition TDM 3D au protocole.

NEMOSIS considère le signal respiratoire comme régulier. Hors d'après la figure 1, la respiration n'est pas reproductible à cause du facteur «stress » du patient. Ce stress est plus important sur la table de traitement, ce qui augmente de façon significative les incertitudes au niveau des phases respiratoires. De plus, notre méthode attribue, pour une phase donnée, toujours une même position à un point, alors que dans la réalité cela est peu probable. La définition des phases ellemême devient alors erronée dans le sens où elle dépend de l'analyse a posteriori d'un signal respiratoire auquel une valeur moyenne sera attribuée sur l'échelle des temps (ce qu'effectue le scanner 4D).

Ces constats ouvrent la voie à l'amélioration de notre plateforme. Ainsi, une nouvelle configuration au niveau des entrées du RNA doit être envisagée pour répondre à la fois à la problématique de la phase qu'au calcul temps réel. Celles-ci sont au nombre de 6 :

- les coordonnées $X_{t}, Y_{t} \%$ et $Z_{t} \%$ du point à simuler exprimées à un instant «t» du signal respiratoire,

- le volume pulmonaire du patient,

- l'amplitude du signal respiratoire à l'instant « $t »$ et $« t+1 »$. 


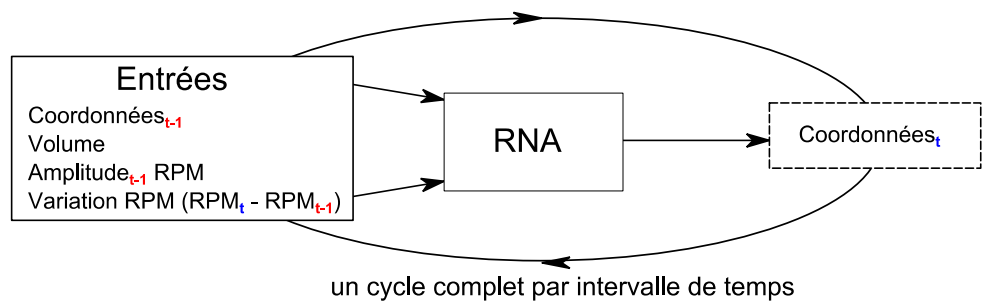

Figure 4 - Schéma de l'évolution de NEMOSIS en réseau bouclé.

Diagram of the evolution of NEMOSIS in a recursive neural network.

Le signal respiratoire étant propre à chaque patient, ces dernières entrées seront importantes dans la personnalisation de la simulation. De plus, ceci permettra de suivre l'évolution en temps réel du signal selon sa fréquence d'enregistrement. Afin de considérer ces variations en temps réel, le type de réseau doit également être adapté. Les réseaux de neurones bouclés (Fig. 4) permettent de réinjecter en entrées des sorties précédemment calculées tout en gardant la structure du réseau initial intact. Toutes les variations de la respiration seront donc considérées et les coordonnées seront adaptées en conséquence.

\section{Conclusion}

NEMOSIS est une plate-forme de simulation de mouvement pulmonaire qui permet de prédire, à partir de coordonnées de point tracé sur une seule image patient (actuellement la phase $0 \%$ définie par le TDM 4D), les coordonnées à toutes les phases respiratoires. Les avantages de notre méthode se situent principalement dans le cadre d'une radiothérapie en temps réel ou lors de l'utilisation d'image 4D dans la planification de traitement. En effet, NEMOSIS n'est pas soumis aux artefacts de l'imageur: cette plate-forme propose des coordonnées différentes d'une phase à une autre. Elle n'interpole pas d'image et simule uniquement la position de points à chaque instant. Elle peut donc s'appliquer à la simulation de contours tumoraux.

Les résultats sur des points anatomiques ont mis en évidence des écarts entre les points tracés et simulés inférieurs à l'ensemble des incertitudes considérées. L'analyse des contours tumoraux générés a donné une bonne concordance (indice de DICE entre 0,80 et 0,97 ) avec ceux tracés par le radiothérapeute. Enfin, les temps de calcul inférieurs à $12 \mathrm{~ms}$ pour générer une phase ont montré qu'une utilisation en temps réel de notre méthode est envisageable. 
L'apport pour le patient n'est pas négligeable: en l'état actuel, seule une acquisition scanner 3D est nécessaire pour déterminer le mouvement sur l'ensemble des phases respiratoires, réduisant ainsi la dose en imagerie. En outre, selon les travaux de Simon (2006), un examen TDM 4D délivre une dose 5 à 7 fois supérieures à un examen TDM 3D classique. L'utilisation de NEMOSIS réduirait donc le bilan dosimétrique d'un patient de ces mêmes facteurs tout en apportant une meilleure connaissance du mouvement. L'utilisation de la plate-forme de simulation à partir d'un TDM 3D classique fera l'objet de travaux ultérieurs.

La détermination numérique du mouvement pulmonaire est un enjeu important dans l'avenir des traitements des tumeurs pulmonaires. En effet, la connaissance du mouvement permet d'améliorer la précision des traitements par la réduction des marges tumorales (ITV et PTV). De plus, dans l'application de notre méthode à l'ensemble du thorax, le développement de NEMOSIS devra se focaliser sur d'autres organes et sur la simulation d'autres mouvements physiologiques tels que les battements cardiaques.

Remerciements. Nous remercions le Pays de Montbéliard Agglomération (PMA), la Ligue Nationale Contre le Cancer (LNCC) ainsi que l'INSERM pour leur soutien financier.

\section{RÉFÉRENCES}

Boldea V. (2006) Intégration de la respiration en radiothérapie : apport du recalage déformable d'images, Thèse de doctorat en informatique, Université Lumière Lyon 2.

Davies S.C., Hill A.L., Holmes R.B., Halliwell M., Jackson P.C. (1994) Ultrasound quantitation of respiratory organ motion in the upper abdomen, Br. J. Radiol. 67 (803), 1096-1102.

Ehrhardt J., Werner R., Säring D., Frenzel T., Lu W., Low D.A., Handels H. (2007) An optical flow based method for improved reconstruction of 4DCT data sets acquired during free breathing, Medical Physics 34 (2), 711-721.

Eom J., Xu X.G., De S., Shi C. (2010) Predictive modeling of lung motion over the entire respiratory cycle using measured pressure-volume data, 4DCT images, and finite-element analysis, Med. Phys. 37 (8), 4389-4400.

Ford E.C., Mageras G.S., Yorke E., Rosenzweig K.E., Wagman R., Ling C.C. (2002) Evaluation of respiratory movement during gated radiotherapy using film and electronic portal imaging, Int. J. Radiat. Oncol. Biol. Phys. 52 (2), 522-531.

Giraud P., De Rycke Y., Dubray B., Helfre S., Voican D., Guo L., Rosenwald J.C., Keraudy K., Housset M., Touboul E., Cosset J.M. (2001) Conformal radiotherapy (CRT) planning for lung cancer: analysis of intrathoracic organ motion during extreme phases of breathing, Int. J. Radiat. Oncol. Biol. Phys. 51 (4), 1081-1092.

Hanley J., Debois M.M., Mah D., Mageras G.S., Raben A., Rosenzweig K.E., Mychalczak B., Schwartz L.H., Gloeggler P.J., Lutz W., Ling C.C., Leibel S.A., Fuks Z., Kutcher G.J. (1999) Deep inspiration breath-hold technique for lung tumors: the potential value of target immobilization and reduced lung density in dose escalation, Int. J. Radiat. Oncol. Biol. Phys. 45 (3), 603-611. 
Hostettler A., Nicolau S.A., Forest C., Soler L., Rémond Y. (2006) Real-time simulation of organ motions induced by breathing: First evaluation on patient data. In: ISBMS, Vol. 4072 of LNCS, pp. 9-18.

Hostettler A., Nicolau S., Soler L., Rémond Y., Marescaux J. (2008) A real-time predictive simulation of abdominal organ positions induced by free breathing. In: Biomedical Simulation, Springer Berlin / Heidelberg (Bello F., Edwards P., Eds), Vol. 5104 of LNCS, pp. 89-97.

Johnston E., Diehn M., Murphy J.D., Loo Jr B.W., Maxim P.G. (2011) Reducing 4DCT artifacts using optimized sorting based on anatomic similarity, Med. Phys. 38 (5), 2424-2429.

Laurent R., Henriet J., Gschwind R., Makovicka L. (2010) A morphing technique applied to lung motions in radiotherapy: preliminary results, Acta Polytechnica 50 (6), 57-65.

Laurent R., Henriet J., Salomon M., Sauget M., Nguyen F., Gschwind R., Makovicka L. (2011) Simulation of lung motions using an artificial neural network, Cancer Radiothérapie 15 (2), 123-129.

Laurent R., Henriet J., Salomon M., Sauget M., Gschwind R., Makovicka L. (2012) Respiratory lung motion using an artificial neural network, Neural Comput. Applic. 21 (5), 929-934, DOI:10.1007/s00521-011-0727-y.

Liu H.H., Balter P., Tutt T., Choi B., Zhang J., Wang C., Chi M., Luo D., Pan T., Hunjan S., Starkschall G., Rosen I., Prado K., Liao Z., Chang J., Komaki R., Cox J.D., Mohan R., Dong L. (2007) Assessing respiration-induced tumor motion and internal target volume using four-dimensional computed tomography for radiotherapy of lung cancer, Int. J. Radiat. Oncol. Biol. Phys. 68 (2), 531-540.

Louie A.V., Rodrigues G., Olsthoorn J., Palma D., Yu E., Yaremko B., Ahmad B., Aivas I., Gaede S. (2010) Inter-observer and intra-observer reliability for lung cancer target volume delineation in the 4D-CT era, Radiother. Oncol. 95 (2), 166-171.

Low D.A., Parikh P.J., Lu W., Dempsey J.F., Wahab S.H., Hubenschmidt J.P., Nystrom M.M., Handoko M., Bradley J.D. (2005) Novel breathing motion model for radiotherapy, Int. J. Radiat. Oncol. Biol. Phys 63 (3), 921-929.

Low D.A., Nystrom M., Kalinin E., Parikh P., Dempsey J.F., Bradley J.D., Mutic S., Wahab S.H., Islam T., Christensen G., Politte D.G., Whiting B.R. (2003) A method for the reconstruction of fourdimensional synchronized CT scans acquired during free breathing, Med. Phys. 30 (6), 12541263

Murphy M.J., Martin D., Whyte R., Hai J., Ozhasoglu C., Le Q.T. (2002) The effectiveness of breathholding to stabilize lung and pancreas tumors during radiosurgery, Int. J. Radiat. Oncol. Biol. Phys. 53 (2), 475-482.

Persson G.F., Nygaard D.E., Brink C., Jahn J.W., Rosenschöld P.M., Specht L., Korreman S.S. (2010) Deviations in delineated GTV caused by artefacts in 4DCT, Radiother. Oncol. 96 (1), 61-66.

Redmond K.J., Song D.Y., Fox J.L., Zhou J., Rosenzweig C.N., Ford E. (2009) Respiratory motion changes of lung tumors over the course of radiation therapy based on respiration-correlated fourdimensional computed tomography scans, Int. J. Radiat. Oncol. Biol. Phys. 75 (5), 1605-1612.

Rietzel E., Pan T., Chen G.T.Y. (2005) Four-dimensional computed tomography: Image formation and clinical protocol, Med. Phys. 32 (4), 874-889.

Sarker J., Chu A., Mui K., Wolfgang J.A., Hirsch A.E., Chen G.T.Y., Sharp G.C. (2010) Variations in tumor size and position due to irregular breathing in 4D-CT: A simulation study, Med. Phys. 37, $3,1254-1260$

Simon L. (2006) Etude comparative et mise en œuvre clinique de deux systèmes de radiothérapie asservie à la respiration, Thèse de doctorat de physique radiologique et médicale, Université de Paris XI - Faculté de Médecine de Paris-Sud. 
Vandemeulebroucke J., Sarrut D., Clarysse P. (2007) The popi-model, a point-validated pixel-based breathing thorax model. In: XVth International Conference on the Use of Computers in Radiation Therapy (ICCR 2007), Toronto, Canada, 4-7 June.

Vandemeulebroucke J., Rit S., Kybic J., Clarysse P., Sarrut D. (2011) Spatiotemporal motion estimation for respiratory-correlated imaging of the lungs, Med. Phys. 38 (1), 166-178.

Van de Steene J., Van den Heuvel F., Bel A., Verellen D., De Mey J., Noppen M., De Beukeleer M., Storme G. (1998) Electronic portal imaging with on-line correction of setup error in thoracic irradiation: clinical evaluation, Int. J. Radiat. Oncol. Biol. Phys. 40 (4), 967-976.

Villard P.F. (2006) Simulation du Mouvement Pulmonaire pour un Traitement Oncologique, Thèse de doctorat en informatique $\mathrm{n}^{\circ} 165-2006$, Université Claude Bernard.

Yamamoto T., Langner U., Loo Jr B.W., Shen J., Keall P.J. (2008) Retrospective analysis of artifacts in four-dimensional CT images of 50 abdominal and thoracic radiotherapy patients, Int. J. Rad. Oncol. Biol. Phys. 72 (4), 1250-1258.

Yang D., Lu W., Low D.A., Deasy J.O., Hope A.J., El Naqa I. (2008) 4DCT motion estimation using deformable image registration and 5D respiratory motion modeling, Med. Phys. 35 (10), 45774590.

Zeng R., Fessler J.A., Balter J.M., Balter P.A. (2008) Iterative sorting for four dimensional CT images based on internal anatomy motion, Med. Phys. 35 (3), 917-926.

Zhao T., Lu W., Yang D., Mutic S., Noel C.E., Parikh P.J., Bradley J.D., Low D.A. (2009) Characterization of free breathing patterns with 5D lung motion model, Med. Phys. 36 (11), 5183-5189. 